\title{
Die kutanen Porphyrien
}

\section{E. Köstler}

U. Wollina

\section{Zusammenfassung}

Die Porphyrien sind Stoffwechselstörungen, denen ein Defekt jeweils eines der Enzyme der Häm- bzw. Porphyrinbiosynthese zugrunde liegt. Sechs der acht Entitäten haben Hautveränderungen, so dass deren Kenntnis diagnostisch wegweisend ist. Die mit schweren Hautveränderungen der freigetragenen Partien einhergehende kongenitale erythropoetische Porphyrie und die klinisch ähnliche hepatoerythropoetische Porphyrie zählen zu den sehr seltenen Erkrankungen. Die erythropoetische Protoporphyrie (Häufigkeit 1:100000) ist in die Differenzialdiagnose akuter Lichtdermatosen stets einzubeziehen. Zur Porphyria cutanea tarda ( $1 \%$ o der Erwachsenenbevölkerung) gibt es neue Erkenntnisse hinsichtlich auslösender oder begleitender Erkrankungen (HCV, HFE-Gene) und zum differenzierten Einsatz der Aderlass- bzw. Chloroquintherapie. Die hereditäre Koproporphyrie und die Porphyria variegata sind in Deutschland selten und können sowohl PCT-ähnliche Hautveränderungen als auch gastrointestinale Krisen und neuropsychiatrische Symptome aufweisen. Die Diagnose ergibt sich primär aus den Stoffwechselmetaboliten-Konstellationen im Urin, im Stuhl und im Blut.

\section{Cutaneous Porphyrias}

\section{Abstract}

Porphyrias are metabolic disorders caused by defects of enzymes of the hem- or porphyrin synthesis. Six out of the eight known diseases also have cutaneous manifestations which may be of diagnostic relevance. Very rare disorders are congenital erythropoietic porphyria and hepatoerythropoietic porphyria which resemble each other. Both are associated with severe skin lesions in the sun-exposed areas. Erythropoietic protoporphyria (incidence $1: 100,000$ ) has always to be considered in differential diagnosis of light-induced skin diseases. There are new insights in trigger factors and associated disorders of porphyria cutanea tarda (PCT) ( 1\% of the adult population affected) like hepatitis C virus infection or HFE-gene mutations that may have an impact on treatment regimens like phlebotomy and/or chloroquine. Hereditary coproporphyria and porphyria variegata are rare in Germany. They may cause PCT-like skin lesions, neuropsychiatric and gastrointestinal symptoms. The diagnosis of porphyria is primarily based on pattern recognition of metabolites in urine, faeces and blood.

\section{Einleitung}

Den Porphyrinkrankheiten liegt eine enzymatische Störung der Synthese der Porphyrinogene und des Häms zu Grunde. Nach dem primären Expressionsort des Enzymdefektes können die Porphyrien in erythropoetische und hepatische Formen unterteilt werden [1], aus klinischer Sicht wird zunehmend in akute und nicht akute Porphyrien klassifiziert [2-4] (Tab.1).
Die nicht akuten Porphyrien sind durch Hautsymptome charakterisiert, bei der PV und HK können zusätzlich zu abdominellen, neurologischen und psychischen Symptomen Hautveränderungen auftreten, die AIP und die Doss-Porphyrie weisen ausschließlich Allgemeinsymptome auf. 
Tab. 1 Klassifikation der Porphyrien

\begin{tabular}{ll}
\hline \multicolumn{1}{l}{ akute Porphyrien } & nicht akute Porphyrien \\
\hline $\begin{array}{l}\text { akute intermittierende } \\
\text { Porphyrie (AIP) }\end{array}$ & $\begin{array}{l}\text { kongenitale erythropoetische } \\
\text { Porphyrie (CEP) }\end{array}$ \\
\hline Porphyria variegata (PV) & erythropoetische Protoporphyrie (EPP) \\
\hline hereditäre Koproporphyrie (HK) & Porphyria cutanea tarda (PCT) \\
\hline Doss-Porphyrie & hepatoerythropoetische Porphyrie (HEP) \\
\hline
\end{tabular}

\section{Kongenitale erythropoetische Porphyrie Günther (CEP)}

J. H. Schultz beschreibt in seiner Greifswalder Dissertation des Jahres 1874 die Krankheit eines Webergesellen aus dem Erzgebirge als „Ein Fall von Pemphigus leprosus complicirt durch Lepra visceralis“. Das ist die erste klinische Darstellung einer Porphyrie, die als kongenitale erythropoetische Porphyrie anzusehen ist [5]. Fritsch et al. haben 1997/98 [6,7] etwa 150 Falldarstellungen aus der Literatur zusammengestellt. Die Erkrankung beruht auf einem homozygoten oder verbunden heterozygoten Defekt der Uroporphyrinogen-III-Cosynthase mit bisher mehr als 20 bekannten Mutationen [8,9]. Als Folge kommt es zur Akkumulation von Porphyrinen der Isomerenreihe I in Körperflüssigkeiten und Organen.

\section{Klinik}

Die ausgeprägte Photosensibilität führt im ersten Lebenssommer zu schmerzhaften blasigen Erythemen. Die Kinder schreien bei Lichtexposition. Die Windeln sind bräunlich-rot verfärbt. Ulzerationen, Narbenbildungen besonders im Kopfbereich, Dyspigmentierungen, Hypertrichosen des Gesichts und der Arme, Osteolysen und Mutilationen an Fingern, Nase und Ohrmuscheln treten hinzu. Die Zähne zeigen infolge eingelagerter Porphyrine eine Erythrodontie. Hornhautulzerationen führen zu Sehbeeinträchtigungen. Hämolytische Anämie mit z.T. monströser Splenomegalie ist häufig vorhanden. Die Lebenserwartung ist mehrheitlich verkürzt $[1,8,10,11]$.

\section{Diagnostik}

Nachweis der extremen Akkumulation von Porphyrinen der Isomerenreihe I im Urin bei normaler Porphyrinvorläuferausscheidung, 10 - 30fache Vermehrung der Porphyrine in den Erythrozyten mit Dominanz des Protoporphyrins $[1,10,12]$.

\section{Therapie}

Eine kausale Therapie gibt es nicht. Neben Lichtschutzmaßnahmen werden Aktivkohle und Cholestyramin verabreicht. Splenektomie mit Transfusionen führen zur vorübergehenden Besserung. Stammzelltransplantationen sind erfolgreich, aber auch mit letalen Ausgängen durchgeführt worden $[2,6,10,13]$. Pränatale biochemische- und Gendiagnostik ist möglich [9].

\section{Erythropoetische Protoporphyrie (EPP)}

Gray beschrieb 1926 eine Lichtdermatose, die er 1964 nachuntersuchte und als EPP klassifizierte [14], nachdem 1961 von Langhof et al. [15] und Magnus et al. [16] unabhängig voneinander die EPP biochemisch und klinisch charakterisiert wurde.

Die Erkrankung beruht auf einem Defekt der Ferrochelatase. Sie wird autosomal dominant mit inkompletter Penetranz vererbt, wiederholt finden sich Berichte über rezessive Erkrankungen; Erklärungen hierfür liefern neuere molekulargenetische Erkenntnisse zum zusätzlichen Genpolymorphismus [17]. Die Häufigkeit beträgt ca. 1:100 000 [18]. Nur in etwa 10\% der Fälle wird eine Weitergabe der elterlichen klinischen Erkrankung auf die Kinder beobachtet [19].

\section{Klinik}

Bereits in frühester Kindheit tritt nach unterschiedlich langer Sonnenexpositionsdauer eine gelegentlich purpurische Rötung mit Schwellung der exponierten Hautareale ein. Folgend juckt und schmerzt die Haut heftig mit nächtlichem Schreien und Weinen sowie dem Drang nach kühlenden Umschlägen. Nach und nach verdickt sich die Haut insbesondere der Handrücken und des Gesichts, die Nase zeigt ein Apfelsinenschalen-Phänomen (Abb.1,2). Etwa 5\% der Patienten entwickeln im frühen Erwachsenenalter eine Leberzirrhose mit Cholestase bis hin zum Leberausfallkoma und seltener Neuropathie (Abb.3). Eine milde Anämie bei niedrigem Serumeisen haben ca. $25 \%$ der EPP-Betroffenen, Gallensteine wurden wiederholt nachgewiesen $[2,10,19-22]$.

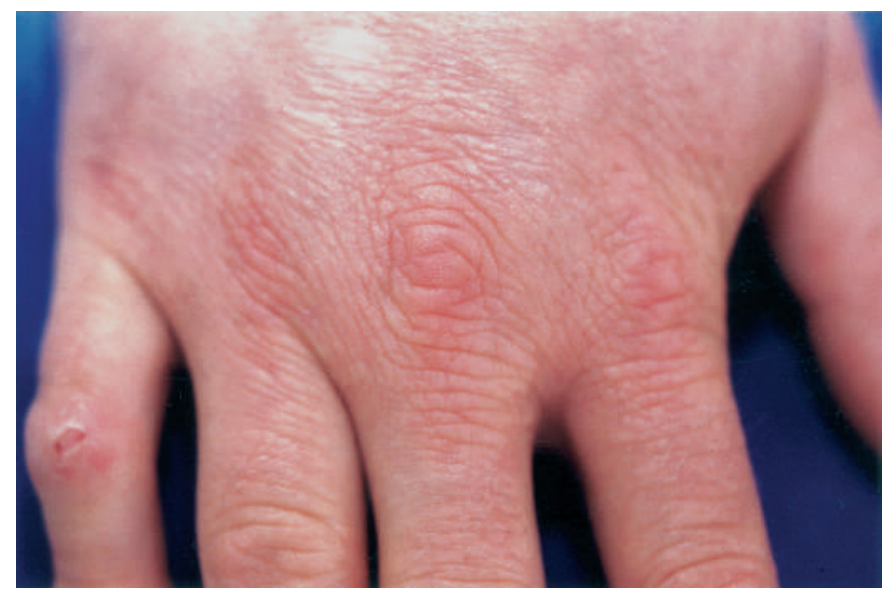

Abb. 1 Erythropoetische Protoporphyrie. Persistierende Hautveränderungen. Vergröbert-verdickte Haut der Handrücken.

\section{Diagnostik}

Das freie Protoporphyrin in den Erythrozyten ist bis auf das 50fache erhöht, dies ist diagnostisch beweisend. Eisenmangel und Bleiintoxikationen führen in der Regel zu geringem Anstieg des Zinkprotoporphyrins. Im Stuhl ist ebenfalls ein erhöhter Protoporphyringehalt nachweisbar $[1,10,11]$. 


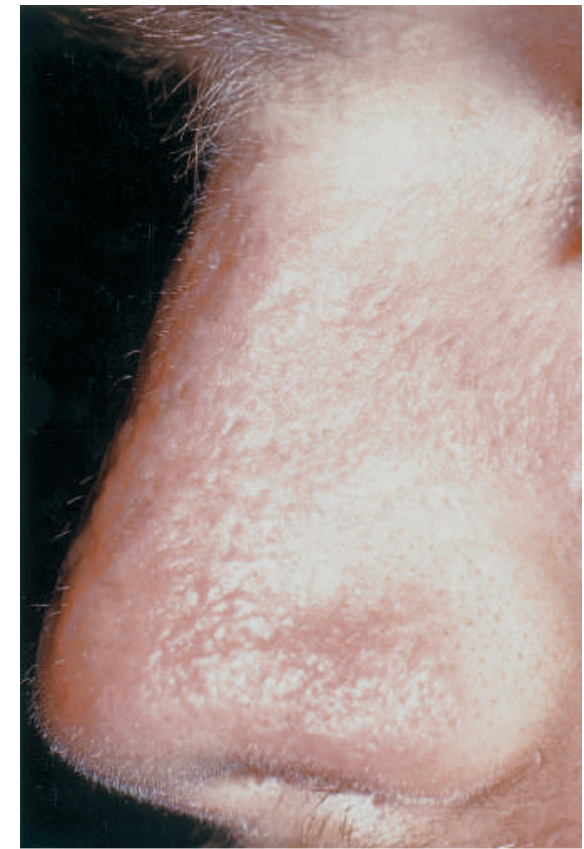

Abb. 2 Erythropoetische Protoporphyrie. Wachsartig, ungleichmäßig-verdicktes Relief des Nasenrückens.

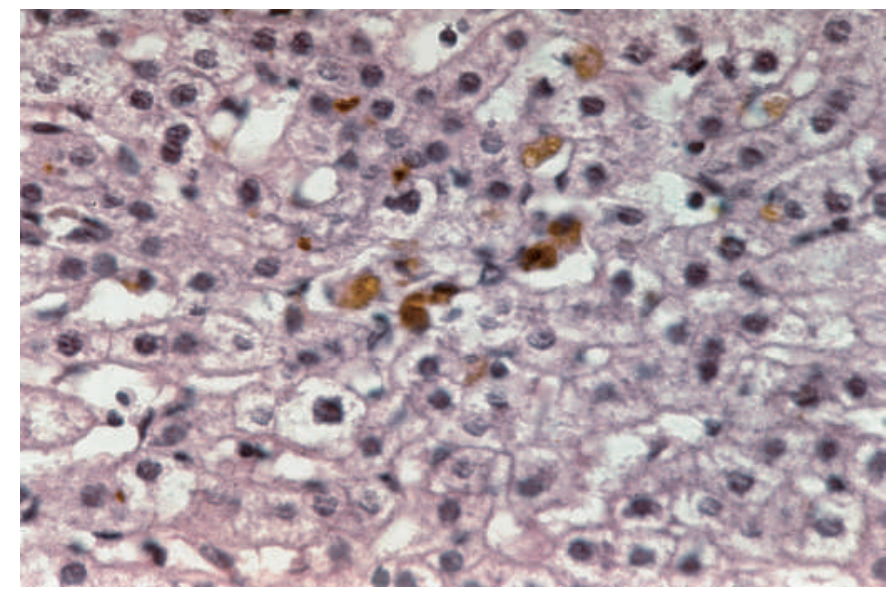

Abb. 3 Erythropoetische Protoporphyrie. Leberhistologie: braune Pigmentschollen in Hepatozyten.

\section{Therapie}

Betakarotin führt bei einem Großteil zur erhöhten Lichttoleranz [10]. Raucher haben unter dieser Medikation jedoch ein erhöhtes Lungenkrebsrisiko [23], Cystein wurde ebenfalls erfolgreich eingesetzt [24]. Von Nutzen sind UVA-undurchlässige gelbe Folien und die modernen Sonnenblocker-Cremes. Ersteres ist besonders bei langdauernden chirurgischen Eingriffen zu beachten. Zur Behandlung protoporphyrininduzierter Lebererkrankungen werden Cholestyramin, Vitamin E und Chenodesoxycholsäure empfohlen [1,2,19-21]. Bis zum Jahr 2000 sind als Ultima Ratio 16 Patienten lebertransplantiert worden [25].

\section{Porphyria cutanea tarda (PCT)}

Die Erstbeschreibung als „chronische Haematoporphyrie“ ist von Günther 1911 [26]. Grundlage der Erkrankung ist eine Defizienz der Uroporphyrinogen-Dekarboxylase (Uro-D), die angeboren (Typ-II-PCT) oder annehmbar erworben (Typ-I-PCT) sein kann.
Letztere sporadische PCT zeigt den Defekt ausschließlich in der Leber, die Typ-II-PCT hat eine ca. 50\% erniedrigte Uro-D-Aktivität in allen Geweben und wird autosomal dominant vererbt. Daneben gibt es auch nach eigenen Beobachtungen einen (autosomal dominanten?) familiären Typ III ohne Uro-D-Aktivitätsminderung in den Erythrozyten [19,26]. Häufigkeitsangaben zur PCT schwanken zwischen $1: 1000$ [27] bis $1: 70000$ [2], es überwiegen Männer zwischen dem 4. und 6. Lebensjahrzehnt [26].

Die Assoziation der PCT zu HFE-Gen-Mutationen bzw. zur Hämochromatose ist für den euro-amerikanischen Raum gesichert [28]. Die PCT ist stets mit einem Leberschaden assoziiert, der von unspezifischen Veränderungen bis hin zur kompletten Zirrhose reichen kann. Glukosestoffwechselstörungen, die Dupuytrensche Kontraktur und Autoimmunerkrankungen (Lupus erythematodes) treten ebenfalls gehäuft auf. Die Hepatitis-C-Infektionshäufigkeit liegt in einem Nord-Süd-Gefälle zwischen 8 und 90\%. Weitere infektiöse Präzipitationsfaktoren wie HI-, Hepatitis-B- und Zytomegalie-Viren sind von regional unterschiedlicher, in unserem Bereich von eher geringer Bedeutung. Andere, die Manifestation der PCT-Typen begünstigende Faktoren sind Alkohol, Östrogene, Eisentherapie, die chronische Hämodialyse bei Niereninsuffizienz und eventuell der Tabakkonsum $[26,29-31]$.

\section{Klinik}

Das stets vorhandene führende klinische Symptom ist eine erhöhte Hautverletzlichkeit lichtexponierter Areale zumeist mit Blasenbildung in diesem Bereich, hinzu kommt eine faziale betont periorbikuläre Hypertrichose bei schiefergrauem Teint. Weiter wurden beobachtet die Skleroporphyrie, eine Alopecia porphyrica, zentrofaziale papulöse Lymphangiektasien, purpurische Veränderungen, subunguale Hämatome, Onycholysen $[10,26]$ sowie neuerdings ein erneutes Dunkelwerden ergrauter Haare [32] (Abb. 4-6). Der Blasenbildung gehen in etwa der Hälfte der Fälle juckend-brennende Sensationen voraus [26], so dass über Fehldiagnosen eines chronischen Handekzems berichtet wurde [33]. Nach Abheilung können Pseudo-Milien auftreten.

Typische Schleimhautveränderungen gibt es nicht. Die „Lackzunge" gilt als Zeichen einer assoziierten Leberzirrhose, beobachtet wurde eine persistierende Schwellung und Rötung der Unterlippe wie bei akuter aktinischer Cheilitis trotz Innenraumberufes [34]. Etwa 50\% der PCT-Patienten haben eine chronische Konjunktivitis, Einzelbeobachtungen betreffend eine Sklerenund Keratomalazie [35].

Einige Untersuchungen weisen ein erhöhtes Leberkarzinomrisiko für PCT-Patienten nach, dies ist jedoch nicht unwidersprochen, offenbar kann diese Komplikation durch eine suffiziente Therapie weitgehend verhindert werden [36-39].

\section{Diagnostik}

5- bis 50fache Vermehrung der Porphyrine im Urin, wobei die hochkarboxylierten Uro- und Heptakarboxyporphyrine überwiegen. Die Porphyrinvorläufer sind im Normbereich, ebenso die Erythrozytenporphyrine. Im Stuhl (Untersuchung nicht erforderlich) ist Isokoproporphyrin nachweisbar. Ergänzt werden diese Untersuchungen durch die Leberdiagnostik, Untersuchung der Eisenparameter und des HFE-Gen-Status (Früherkennung der 


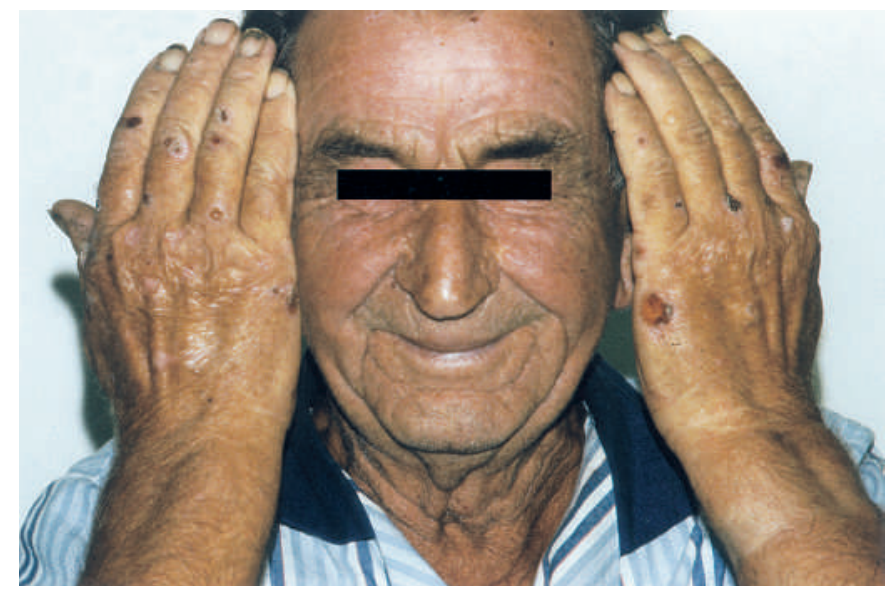

Abb. 4 Porphyria cutanea tarda. Dunkle Gesichtsfarbe, periorbikuläre Hypertrichose; Erosionen, Krusten, Narben und Blasen der Handrücken.

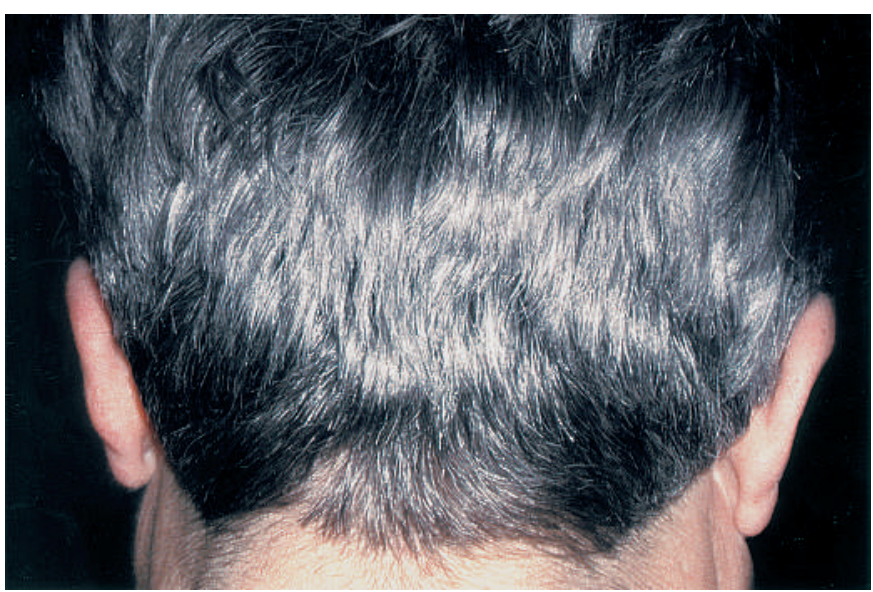

Abb. 5 Porphyria cutanea tarda. Dunklere Haare des Nackenhaaransatzes (waren vor der Erkrankung grau).

Hämochromatose) sowie den Ausschluss möglicher begleitender Stoffwechselstörungen (insbesondere des Diabetes mellitus) $[1,10,26,28]$.

\section{Therapie}

Die Therapie der PCT beinhaltet die Ausschaltung von Triggerfaktoren, die Aderlassbehandlung und/oder Chloroquingaben.

Die Mehrzahl der Triggerfaktoren können leberschädigend wirken und sollten allein deshalb gemieden werden. Ihre Ausschaltung kann zu latenten Krankheitszuständen führen [40]. Da die Leberveränderungen langbestehender unbehandelter PCT eine Verschlechterungstendenz haben, sollte stets eine sanierende Therapie angestrebt werden [26].

Die eisenentziehende Aderlassbehandlung nach Ippen $(500 \mathrm{ml}$ Aderlass wöchentlich über 4 Wochen, $500 \mathrm{ml}$ 14-tägig oder monatlich bis zur Normalisierung der Porphyrinausscheidung) ist klinisch sicher wirksam, hat Kontraindikationen wie vorbestehende Anämie, Eiweißverlust bei Leberzirrhose und ist aufwändig, da nicht arztfern.

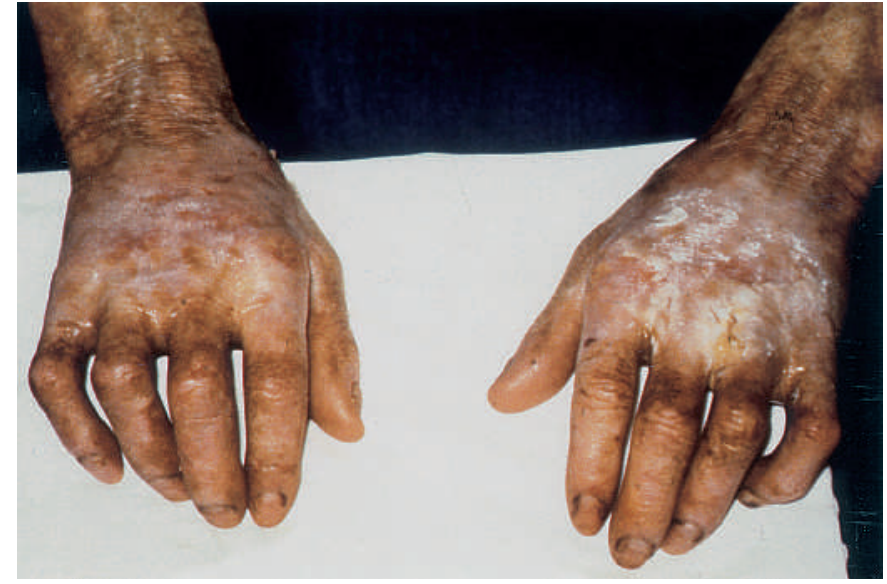

Abb. 6 Porphyria cutanea tarda. Massive sklerodermiforme Hautveränderungen der Hände mit Bewegungseinschränkungen.

Zur Elimination der Porphyrine hat sich die Chloroquintherapie in niedrig dosierter intermittierender Anwendungsweise, $2 \times$ wöchentlich 125 ( $-250 \mathrm{mg}$ ) ca. 1 Jahr lang, etabliert. Jüngst wurde nachgewiesen, dass bei homozygoten-HFE-Gen-Mutationen diese Therapie versagen kann bzw. durch rasche Rezidive belastet ist. Zudem tritt eine signifikante Verminderung der Eisenspeicher nur bei HFE-Gen-Wildtypträgern ein. Daher ist bei ersterer Patientengruppe die Aderlasstherapie zu präferieren [28]. Beide Behandlungsmaßnahmen können kombiniert werden [41]. In hepatohistologischen Verlaufsstudien konnte eine Besserung des Leberschadenbildes bei $70 \%$ der mit Chloroquin therapierten Patienten aufgezeigt werden $[26,42]$.

Der Sonderfall der hämodialyse-assoziierten Porphyrie kann mit Erythropoetingaben und gleichzeitigen kleinvolumigen Aderlässen behandelt werden, Chloroquin versagt.

Selbst wenn posttherapeutisch die quantitative Porphyrinausscheidung im Normbereich ist, bleiben mehrheitlich Veränderungen der Fraktionenprofile bestehen [42,44]. Rezidive können in bis zu einem Drittel der Fälle auftreten.

\section{Hepatoerythropoetische Porphyrie (HEP)}

Simon et at. stellten 1967 eine ungewöhnlich schwere familiäre PCT vor, die nachfolgend als HEP diagnostiziert wurde [45]. Zwischenzeitlich gibt es ca. 40 Fallbeschreibungen der HEP, die Elder et al. 1981 [46] als homozygote (oder verbunden heterozygote) PCT erkannt haben. Die Erkrankung wird rezessiv vererbt, die Uro-D-Aktivität kann $<10 \%$ des Normwertes betragen. Das klinische Bild entspricht weitgehend der CEP, die urinporphyrinanalytischen Befunde dagegen der PCT. Allerdings sind, offensichtlich mit unterschiedlichen Genotypen zusammenhängend, milde klinische Verlaufsformen bekannt [47]. Im Gegensatz zur PCT ist das Erythrozytenprotoporphyrin (zinkchelatiert) ebenfalls, wenn auch nur mäßig, erhöht [47]. Lebermitbeteiligung unterschiedlichen Ausmaßes wird beschrieben [26]. Eine effektive Therapie ist nicht bekannt, Photoprotektion wird empfohlen. Pränatale Diagnostik der HEP ist durchgeführt worden [48]. 
Diese 1928 von Hijmas van den Bergh [49] beschriebene seltene akute Porphyrie hat in $20 \%$ der Fälle neben den Allgemeinsymptomen der akuten Porphyrie der PCT vergleichbare Hautveränderungen [19]. Die autosomal dominante Erkrankung beruht auf Punktmutationen des Koproporphyrinogenoxidase-Gens.

Entsprechend den übrigen akuten Porphyrien können präzipitierende Faktoren wie verschiedene Medikamente, Infektionen, Hunger, Stress, Menstruation und Alkohol die akuten Attacken auslösen. Die neurologischen und abdominellen Beschwerden schließen abdominelle Schmerzen und Krämpfe, Erbrechen, Diarrhö, Muskelschwäche, Depressionen und Halluzinationen sowie hypertone Kreislaufsensationen mit Tachykardie ein $[19,50]$. Die Erhöhung der Porphyrinvorläufer im Urin während der akuten Attacken und die starke Erhöhung der Koproporphyrine in Urin und Stuhl sind diagnostisch wegweisend $[1,50]$.

Zur Therapie der akuten Porphyrien sei auf die Zusammenstellung in der „Roten Liste“ verwiesen [51].

\section{Porphyria variegata (PV)}

Die Erstbeschreibung geht auf Hijmas van den Bergh und Gotepass [52] im Jahre 1937 zurück. Die Erkrankung beruht auf einem Defekt der Protoporphyrinogenoxidase, ist autosomal dominant vererbbar und findet sich außerhalb Südafrikas und Chiles (Gründereffekte) nur selten [11,53]. Die Hauterscheinungen sind von denen der PCT nicht zu unterscheiden, treten aber zumeist bereits postpubertär bzw. im frühen Erwachsenenalter auf [53]. Von 50 Patienten mit PV in Südafrika hatten $43 \%$ ausschließlich Hauterscheinungen, 10\% akute Attacken und Hauterscheinungen, 9\% ausschließlich akute Attacken und 38\% waren asymptomatisch [53].

Die Diagnostik erfordert Urin- und Stuhluntersuchungen mit typischen Porphyrinprofilen. Im Urin dominiert Koproporphyrin, Porphyrinvorläufer sind insbesondere während akuter Attacken erhöht. Im Stuhl überwiegt das erhöhte Protoporphyrin das Koproporphyrin [1]. Im Plasma ist bei $626 \mathrm{~nm}$ ein typisches Fluoreszenz-Emissionsmaximum nachweisbar [54].

Die Therapie der akuten Attacken ist bei allen Porphyrieformen gleich [51], bei HK und PV wird zusätzlich Photoprotektion empfohlen [19].

\section{Akute homozygote und duale Porphyrien}

In frühester Kindheit auftretende homozygote oder verbunden heterozygote akute Porphyrien mit schwersten Allgemeinerscheinungen sind kasuistisch beschrieben worden. Sie können zusätzlich mutilierende Hautveränderungen und eine starke Photosensibilität aufweisen [55-57]. Einzelberichte dualer Porphyrien (AIP/PV, AIP/PCT, HK/CEP, PV/PCT) zeigen häufig ebenfalls eine frühzeitige Manifestation mit biochemischen und klinischen Merkmalen beider Entitäten [58,59].
${ }^{1}$ Doss MO. Krankheiten und Störungen der Porphyrin- u. Hämbiosynthese. In: Gross R, Schölmerich P, Gerok W (Hrsg). Die Innere Medizin 10. Aufl. Stuttgart: Schattauer, 2000: 1175-1192

${ }^{2}$ Murphy GM. The cutaneous porphyrias: a review. Br J Dermatol 1999; 140: $573-581$

${ }^{3}$ Doss MO. Hepatische Porphyrien. Symptomatik, Diagnostik und Therapie. internist prax 2004; 44: 797-802

${ }^{4}$ Köstler E. Die Porphyrien. Z ärztl Fortbild Qual sich 1999; 93: 222 223

${ }^{5}$ Helgason E, Latotzki H. Zur Geschichte der Porphyria erythropoetica. Zschr ges inn Med 1965; 20: 759-763

${ }^{6}$ Fritsch C, Bolsen K, Ruzicka T, Goerz G. Congenital erythropoietic porphyria. Clinical review. J Am Acad Dermatol 1997; 36: 594-610

${ }^{7}$ Fritsch C, Nürnberger W, Bolsen K, Lehmann P, Ruzicka T. Kongenitale erythropoetische Porphyrie. Z Hautkr 1998; 73: 247-250

${ }^{8}$ Herrera Saval A, Moruno Tirado A. Congenital erythropoietic porphyria affecting two brothers. Br J Dermatol 1999; 141: 547-550

9 Pannier E, Viot G, Aubry MC, Grange G, Tantau J, Fallet-Bianco C, Müller F, Cabrol D. Congenital erythropoietic porphyria (Günther's disease): two cases with very early prenatal manifestation and cystic hygroma. Prenat Diagn 2003; 23: 25-30

${ }^{10}$ Goerz G, Scharffetter-Kochanek K. Die Porphyrien. Akt Dermatol 1994; 20: $103-113$

${ }^{11}$ Poblete Gutierrez P, Wiederholt T, Frank J. Die Porphyrien: Was Dermatologen wissen sollten. Z Hautkr 2002; 77: 265-275

12 Gross U, Hoffmann GF, Doss MO. Erythropoietic and hepatic porphyrias. J Inherit Metab Dis 2000; 23: 641 - 661

${ }^{13}$ Dawe SA, Stephens AD, Peters DJ, du Vivier A, Creamer JD. Congenital erythropoietic porphyria: dilemmas in present day management. Clin Exp Dermatol 2002; 27: 680-683

${ }^{14}$ Gray CH, Kulczycka A, Nicholson DS, Magnus A, Rimington C. Isotope studies on a case of erythropoietic protoporphyria. Clin Sci 1964; 26: $7-15$

${ }^{15}$ Langhof H, Müller H, Rietschel L. Untersuchungen zur familiären protoporphyrinämischen Lichturticaria. Arch Klin Exp Dermatol 1961; 212: $506-518$

${ }^{16}$ Magnus IA, Jarret A, Prankerd TAJ, Rimington C. Erythropoietic protoporphyria: A new porphyria syndrome with solar urticaria due to protoporphyrinaemia. Lancet 1961; II: $448-451$

17 Gouya L, Puy H, Robreau A-M, Burgeois M, La MorilJ, da Silva V, Grandchamp B, Deybach J-Ch. The penetrance of dominant erythropoietic protoporphyria is modulated by expression of wildtype FECH. Nat Genet 2002; 30: $27-28$

${ }^{18}$ Went LN, Klasen EC. Genetic aspects of erythropoietic protoporphyria. Am Hum Genet 1984; 48: 105 - 117

${ }^{19}$ Lim HW, Cohen JL. The cutaneous porphyrias. Sem Cutan Med Surg 1999; 18: 285-292

${ }^{20}$ Nguyen L, Blust M, Bailin M, Melendez L, Raines DE. Photosensitivity and perioperative polyneuropathy complicating orthotopic liver transplantation in a patient with erythropoietic protoporphyria. Anesthesiol 1999; 91: $1173-1175$

${ }^{21}$ Köstler E. Die erythropoetische Protoporphyrie. Dermatol Monatschr 1992; 178: $274-281$

22 Atkins AM, Nedorost ST. Swollen upper extremities. Clin Pediatr 2003; 42: $827-829$

${ }^{23}$ Omenn GS, Goodman GE, Thornquist MD, Balmes J, Cullen MR, Glass A, Keogh JP, Meyskens FL, Valanis B, Williams JH, Barnhart S, Cherniack MG, Brodkin CA, Hammar S. Risk factors for lung cancer and for intervention effects in CARET, the Beta-Carotene and Retinol Efficacy Trial. J Natl Cancer Inst 1996; 88: 1550-1559

${ }^{24}$ Mathews-Roth MM, Rosner B. Longterm treatment of erythropoietic protoporphyria with cysteine. Photodermatol Photoimmunol Photomed 2002; 18: 307-309

${ }^{25}$ Meerman L. Erythropoietic protoporphyria. An overview with emphasis of the liver. Scand J Gastroenterol 2000; 35 Suppl 232: 79-85

${ }^{26}$ Köstler E, Doss MO. Die chronische hepatische Porphyrie (Porphyria cutanea tarda). In: Brandis M, Fanconi A, Frick P, Kochsiek K, Riecken EO (Hrsg). Ergebn Inn Med Kinderheilk. Berlin, Heidelberg, New York: Springer, 1993: $123-205$

${ }^{27}$ Martasek P, Kordac V, Zoubek V, Enchdolgor G, Fekete Z, Bartek J, Goldenberg A, Kotal P. Epidemiology of porphyria cutanea tarda in Cze- 
choslovakia and Mongolia. In: Topi GC, D’Alessandro Gandolfo L (Hrsg). Boll Ist Dermatol S Gallicano 1986/87; 13: 330-331

${ }^{28}$ Stölzel U, Köstler E, Schuppan D, Richter M, Wollina U, Doss MO, Wittekind Ch, Tannapfel A. Hemochromatosis (HFE) gene mutations and response to chloroquine in porphyria cutanea tarda. Arch Dermatol 2003; 139: 309-313

${ }^{29}$ Stölzel U, Schuppan D, Tillmann HL, Manns MP, Tannapfel A, Doss MO, Zimmer T, Köstler E. Autoimmunity and HCV infection in porphyria cutanea tarda: a controlled study. Cell Mol Biol 2002; 48: 43-47

${ }^{30}$ Egger NG, Goeger DE, Payne DH, Miskovsky EP, Weinman STA, Anderson KE. Porphyria cutanea tarda. Multiplicity of risk factors including HFE mutations, hepatitis C, and inherited uroporphyrinogen decarboxylase deficiency. Dig Dis Sci 2002; 47: 419-426

31 Doss MO, Kühnel A, Groß U, Sieg I. Hepatische Porphyrien und Alkohol. Med Klin 1999; 94: $314-328$

32 Shaffrali FCG, Mc Donagh AJG, Messenger AG. Hair darkening in porphyria cutanea tarda. Br J Dermatol 2002; 146: 325-329

${ }^{33}$ Sommer S, Wilkinson STM. Porphyria cutanea tarda masquerading as chronic hand eczema. Acta Derm Venereol 2003; 84: 170 - 171

${ }^{34}$ Martinez A, Morales R, Brethauer U, Jimenez M, Alarcon R. Porphyria cutanea tarda affecting lower lip. Oral Surg Med Oral Pathol Oral Radiol Endod 2000; 90: 705 - 708

35 Pfeufer E-M. Klinische Untersuchungen zur Augenbeteiligung bei der Porphyria cutanea tarda. Diss Med Fak TU Dresden. Dresden: 1998

${ }^{36}$ Köstler E. Gibt es die paraneoplastische Porphyria cutanea tarda? Dtsch Z Verdauungs-Stoffwechselkr 1988; 48: 35-40

${ }^{37}$ Kordac V, Jirsa M, Kotal P, Kalab M, Cervinka J, Kotyk A, Martasek P. Agents affecting porphyrin formation and secretion: implications for porphyria cutanea tarda treatment. Semin Hematol 1989; 26: 16-23

${ }^{38}$ Fracanzani AL, Taioli E, Sampietro M, Fatta E, Bertelli C, Fiorelli G, Fargion S. Liver cancer risk is increased in patients with porphyria cutanea tarda in comparison to matched control patients with chronic liver disease. J Hepatol 2001; 35: 498 -503

${ }^{39}$ Gisbert JP, Garcia-Buey L, Alonso A, Rubio S, Hernandez A, Pajares JM, Garzia-Diez A, Moreno-Otero R. Hepatocellular carcinoma risk in patients with porphyria cutanea tarda. Eur J Gastroenterol Hepatol 2004; 16: 689-692

40 Topi GC, Amantea A, Griso D. Rocevery from porphyria cutanea tarda with no specific therapy other than avoidance of hepatic toxins. $\mathrm{Br} J$ Dermatol 1984; 111: 75-82

${ }^{41}$ Seubert S, Seubert A, Stella M, Guzman H, Battle A. Ergebnisse bei der Behandlung der Porphyria cutanea tarda mit Aderlaß und Resochin. Z Hautkr 1990; 65: 223-225

42 Köstler E, Rießland M, Seebacher C. Chronische hepatische Porphyrie (Porphyria cutanea tarda): Porphyrinurie und leberorientierte Parameter unter Therapiekontrolle. Lab Med 1992; 16: 35- 39

${ }^{43}$ Poux J-M, Cadranel J-F, Domentis R. Porphyria cutanea tarda, hepatitis C infection, and iron overload in a patients on hemodialysis: The reply. Am J Med 1999; 106: 266-267
${ }^{44}$ To-Figueras J, Ozalla D, Mateu CH. Long-standing changes in urinary profile of porphyria isomers after clinical remission of porphyria cutanea tarda. Ann Clin Lab Sci 2003; 33: 251 - 256

${ }^{45}$ Koszo F, Elder CH, Roberts A, Simon N. Uroporphyrinogen decarboxylase deficiency in hepatoerythropoietic porphyria: further evidence for genetic heterogeneity. Br J Dermatol 1990; 122: 365 - 370

${ }^{46}$ Elder GH, Smith SG, Herrero C, Lecha M, Mascaro JM, Muniesa AM, Czarnecki DB, Brenan J, Poulos V, de Salamanca RE. Hepatoerythropoietic porphyria: a new uroporphyrinogendecarboxylase defect or homozygous porphyria cutanea tarda? Lancet 1981; I: 916-919

${ }^{47}$ Armstrong DKB, Sharpe PC, Chambers CR, Whatley SD, Roberts AG, Elder GH. Hepatoerythropoietic porphyria: a missense mutation in the Uro-D gene is associated with mild disease and an unusual porphyrin excretion pattern. Br J Dermatol 2004; 151: 920-923

${ }^{48}$ Ged C, Ozalla D, Herrero C, Lecha M, Mendez M, de Verneuil H, Mascaro $M$. Description of a new mutation in hepatoerythropoietic porphyria and prenatal exclusion of a homozygous fetus. Arch Dermatol 2002; 138: $957-960$

${ }^{49}$ Hijmans van den Bergh AA, Regniers L, Müller SE. Ein Fall von kongenitaler Porphyrinurie mit Koproporphyrin im Harn und Stuhl. Arch Verdauungskr 1928; 42: $302-307$

${ }^{50}$ Kühnel A, Groß U, Doss MO. Hereditary coproporphyria in Germany: clinical-biochemical studies in 53 patients. Clin Biochem 2000; 33: $465-473$

51 Doss MO, Stölzel U, Doss M. Arzneistoffe bei akuten hepatischen Porphyrien und Empfehlungen zur Anästhesie. In: Rote Liste 2004. Aulendorf: Editio Cantor, 2004: 495-496

${ }^{52}$ Hijmans van den Bergh AA, Gotepass W. Ein bemerkenswerter Fall von Porphyrie. Wien Klin Wochenschr 1937; 50: 830-831

${ }^{53}$ Kirsch R, Meissner P, Hift R. Variegate porphyria. Semin Liver Dis 1998; 18: 33-41

${ }^{54}$ Poh-Fitzpatrick MB. A plasma porphyrin fluorescence marker for variegate porphyria. Arch Dermatol 1980; 116: 543-547

${ }^{55}$ Elder GH. Hepatic porphyrias in children. J Inher Metab Dis 1997; 20: $237-246$

${ }^{56}$ Grandchamp B, Phung N, Nordmann Y. Homozygous case of hereditary coproporphyria. Lancet 1977; II: $1348-1349$

${ }^{57}$ Hift RJ, Meissner PN, Todd G, Kirby P, Bilsland D, Collins P, Ferguson J, Moore MR. Homozygous variegate porphyria: an evolving clinical syndrome. Postgrad Med J 1993; 69: 781 - 786

${ }^{58}$ Doss MO, Groß U, Puy H, Doss M, Kühnel A, Jacob K. Koexistenz von hereditärer Koproporphyrie und Porphyria cutanea tarda: Eine neue Form einer dualen Porphyrie. Med Klin 2002; 97: 1 - 5

${ }^{59}$ Freesemann A, Hofweber K, Doss MO. Coexistence of deficiencies of uroporphyrinogen III synthase and decarboxylase in a patient with congenital erythropoietic porphyria and in his family. Eur J Clin Chem Clin Biochem 1997; 35: 35-39 